\title{
ON A CLASSIFICATION OF GRADIENT SHRINKING SOLITONS
}

\author{
Lei Ni and Nolan Wallach
}

\begin{abstract}
The main purpose of this article is to provide an alternate proof to a result of Perelman on gradient shrinking solitons. Moreover in dimension three our proof generalizes Perelman's result by removing the $\kappa$-non-collapsing assumption and allowing general curvature growth. The method also allows us to prove a classification result on gradient shrinking solitons with vanishing Weyl curvature tensor in high dimensions, which includes the rotationally symmetric ones.
\end{abstract}

\section{Introduction}

In his surgery paper [P2] Perelman proved the following statement (we refer the reader to $[\mathrm{P} 2]$ as well as $[\mathrm{CZ}, \mathrm{KL}, \mathrm{MT}, \mathrm{CLN}]$ for unexplained terminology and notation):

Theorem 1.1 (Perelman). Any three dimensional $\kappa$-non-collapsed gradient shrinking soliton with bounded positive sectional curvature must be compact.

This combined with Hamilton's convergence (or curvature pinching) result [H1] (see also [I]) implies that $M^{3}$ must be isometric to a quotient of $\mathbb{S}^{3}$. The importance of such a result is that it rules out the possible complications caused by the (a priori possible) existence of noncompact singularity models with positive curvature and implies a classification of finite time singularity models. This allows the use of surgery methods possible in the case of dimension three. More precisely, ancient solutions (which are noncompact in interesting cases) can be obtained as the Cheeger-Gromov limit of the sequence of blow-ups (via the compactness result of Hamilton [H3]) as we approach to the singular time. The gradient shrinking solitons arise from the non-collapsed ancient solutions as blow-down limits [P1] (at least) in the case that the ancient solution has nonnegative curvature operator. By ruling out noncompact shrinking solitons with positive curvature one can conclude that a shrinking soliton that arises from an ancient solution must be the cylinder $\mathbb{S}^{2} \times \mathbb{R}$ or a quotient of it. This provides the phototype for the surgery. This relation of the gradient shrinking solitons with the Ricci flow suggests the importance of studying noncompact gradient shrinking solitons.

On the other hand, Perelman's proof, of which has a detailed exposition in [CZ, $\mathrm{KL}, \mathrm{MT}$ ] (see also pages 377-386 of [CLN]), is geometric and relies on detailed analysis of the level sets of the potential function, and more importantly, the Gauss-Bonnet formula for surfaces. The authors could not adapt Perelman's argument to higher

\footnotetext{
Received by the editors September 11, 2007.

The first author's research was supported in part by NSF grant DMS-0504792 and an Alfred P. Sloan Fellowship, USA. The second author's research was partially supported by an NSF grant DMS-0500495.
} 
dimensions. The main goal of this article is to provide an alternate approach and generalize the above result of Perelman to the dimensions greater than 3 . We remove the uniform bound assumption on curvature. Perhaps more importantly we do not assume that the gradient shrinking soliton is $\kappa$-non-collapsed, as assumed in the above mentioned result of Perelman. For the high dimensional case, our method gives a classification of gradient shrinking solitons which are locally conformally flat. The following is our main result.

Theorem 1.2. Let $\left(M^{n}, g\right)$ be a gradient shrinking soliton whose Ricci curvature is nonnegative. If $n \geq 4$ we assume that $(M, g)$ is locally conformally flat. Then either $M$ is isometric to $\mathbb{R}^{n}$, or it is a quotient of $\mathbb{S}^{n}$ or $\mathbb{S}^{n-1} \times \mathbb{R}$. In the case that $M$ is compact, the assumption that the Ricci curvature is nonnegative is not needed.

In particular, if $\left(M^{n}, g\right)$ has positive Ricci curvature it must be compact.

As a corollary we have a more general result than Theorem 1.1.

Corollary 1.3. Let $\left(M^{3}, g\right)$ be a gradient shrinking soliton whose Ricci curvature is positive. Then $M$ must be compact.

It is important to relax the condition of bounded curvature since the gradient shrinking solitons obtained from singularity analysis usually do not have bounded curvature (cf. $[\mathrm{N}]$ ). Secondly, when $M$ is noncompact, the assumption that Ricci curvature is nonnegative is only used to ensure that the integrals involved are finite. 1

Some new invariant cones, which bound the Weyl curvature by the scalar curvature, have been discovered in [BW2] very recently. They may be related to our result.

The rotationally symmetric gradient shrinking solitons has been studied in $[\mathrm{K}]$. A straightforward computation shows that the rotational symmetric manifolds have vanishing Weyl curvature. Hence our result gives a self-contained classification of rotationally symmetric gradient shrinking solitons. (The proof in $[\mathrm{K}]$ appealed the strong result of Böhm-Wilking [BW1] for the compact case.)

\section{Acknowledgements}

The first author would like to thank T. Ilmanen for interests and hospitality during his visit at ETH in the summer of 2007.

\section{Preliminaries}

Recall that $(M, g)$ is a gradient shrinking soliton if there exists a function $f$ such that its Hessian $f_{i j}$ satisfies

$$
R_{i j}+f_{i j}-\frac{1}{2} g_{i j}=0 .
$$

As shown in [CLN], Theorem 4.1, there exists a family of metrics $g(t)$, a solution to Ricci flow with the property that $g(0)=g$ and a family of diffeomorphisms $\phi(t)$, which is generated by the vector field $X=\frac{1}{\tau} \nabla f$, such that $\phi(0)=\mathrm{id}$ and $g(t)=\tau(t) \phi^{*}(t) g$ with $\tau(t)=1-t$, as well as $f(t)=\phi^{*}(t) f$. The following can be checked by some straight forward computations [CLN].

\footnotetext{
${ }^{1}$ In fact, the nonnegativity of the Ricci curvature can be removed in the case. This is a observation due to Petersen and Wylie. Please see the Remark 5.5.
} 
Lemma 2.1. For $\tau>0$,

$$
\begin{aligned}
\frac{\partial}{\partial \tau}|\operatorname{Ric}|^{2} & =-\frac{2}{\tau}|\operatorname{Ric}|^{2}-\left\langle\nabla|\operatorname{Ric}|^{2}, \nabla f\right\rangle, \\
\frac{\partial}{\partial \tau} S^{2} & =-\frac{2}{\tau} S^{2}-\left\langle\nabla S^{2}, \nabla f\right\rangle .
\end{aligned}
$$

Here $S$ is the scalar curvature.

This holds, in particular, at $t=0$ (namely $\tau=1$ ). A direct consequence is that

$$
\frac{\partial}{\partial t}\left(\frac{|\operatorname{Ric}|^{2}}{S^{2}}\right)=\left\langle\nabla\left(\frac{|\operatorname{Ric}|^{2}}{S^{2}}\right), \nabla f\right\rangle .
$$

We shall also need the following results. First we need Proposition 1.1 of $[\mathrm{N}]$ to bound the scalar curvature from below.

Proposition 2.2. Assume that $(M, g)$ is a non-flat gradient shrinking soliton. Assume that it has nonnegative Ricci curvature. Then there exists $\delta=\delta(M)>0$ such that $S \geq \delta$.

The following result on the bound of $f$ as well as its gradient is implicit in the argument of [P2] when the Ricci curvature is assume to be bounded. For the general case without assuming the curvature bound, it is included in the proof of Proposition 1.1 of $[\mathrm{N}]$.

Lemma 2.3. Assume the same assumption as in Proposition 2.2. Then for any $A>0$, there exist constants $B=B(M, f, A), C=C(M, f)>0$ such that

$$
\begin{gathered}
f(x) \geq A r(x)-C, \\
f(x) \leq 2 r^{2}(x), \quad|\nabla f|(x) \leq 4 r(x)
\end{gathered}
$$

for $r(x) \geq B$. Here $r(x)$ is the distance function to some fixed point $o \in M$ with respect to $g(0)$ metric. Moreover

$$
S(x) \leq C(r(x)+1)^{2}
$$

for some $C>0$.

Under the assumption that $(M, g)$ has nonnegative Ricci curvature, it is also an easy consequence of the soliton equation (e.g. from the proof of Proposition 1.1 in $[\mathrm{N}])$ that

$$
|\nabla S|^{2} \leq 4 S^{2}|\nabla f|^{2} .
$$

Using the soliton equation and the assumption that $R_{i j} \geq 0$ we also have that

$$
\left|f_{i j}\right|^{2} \leq \max \left\{\frac{n}{2}, S^{2}\right\} .
$$

We will use these inequalities to justify the finiteness of some integrals. Most importantly recall the following local derivative estimates of Shi (cf. Theorem 13.1 of $[\mathrm{H} 3])$. 
Theorem 2.4. For any $\alpha>0$ there exists a constant $C(n, K, r, \alpha)$ such that if $(M, g(t))$ is a solution to Ricci flow with $t \in\left[0, t_{1}\right], 0<t_{1} \leq \frac{\alpha}{K}, p \in M$ and

$$
\left|R_{i j k l}\right|(x, t) \leq K
$$

for all $x \in B_{g(0)}(p, r), \quad t \in\left[0, t_{1}\right]$, then

$$
\left|\nabla_{s} R_{i j k l}\right|(y, t) \leq \frac{C(n, \sqrt{K} r, \alpha) K}{\sqrt{t}}
$$

for all $y \in B_{g(0)}\left(p, \frac{r}{2}\right)$ and $t \in\left(0, t_{1}\right]$. Moreover, for the same $(y, t)$

$$
\left|\nabla^{m} R_{i j k l}\right|(y, t) \leq \frac{C(n, m, K, r, \alpha)}{t^{\frac{m}{2}}} .
$$

\section{Three dimensional case}

We first give a different proof to Perelman's theorem mentioned in the introduction. In fact what we prove is a more general result since we assume neither that gradient shrinking soliton is $\kappa$-noncollapsed nor that the curvature is uniformly bounded. Most argument of the proof can also be used in dimensions $n \geq 4$.

For the argument involving the integration by parts, we assume that there exists $\beta>0$ the Ricci curvature satisfies that

$$
|\operatorname{Ric}|(y, t) \leq \beta\left(r^{2}(x)+1\right)
$$

for all $y \in B_{g\left(-\frac{1}{2}\right)}\left(x, \frac{r(x)}{2}\right)$ and $t \in\left[-\frac{1}{2}, 0\right]$. Here $r(x)$ is the distance function to some fixed point $o \in M$ with respect to the metric $g(0)$. Notice that (3.1) can be easily verified if we assume that $\mid$ Ric $\mid$ is uniformly bounded at $t=0$. It is also not hard to show that under the assumption Ric $\geq 0$, the assumption (3.1) follows from (2.6). Namely on a gradient shrinking soliton with nonnegative Ricci curvature the assumption (3.1) is always true. The main purpose of this section is to show the following result.

Theorem 3.1. Let $\left(M^{3}, g\right)$ be a complete gradient shrinking soliton with the positive sectional curvature. Then $M$ must be the quotient of $\mathbb{S}^{3}$.

Note that we do not need to assume $(M, g)$ is $\kappa$-non-collapsed. The proof also concludes that $M=\mathbb{S}^{3} / \Gamma$ directly without appealing to Hamilton's result. In later sections we, in fact, directly obtain a classification of solitons under the assumption that Ric $\geq 0$.

First we recall a result of Hamilton. In [H1], the following result was proved for solutions to Ricci flow on a three manifold $M$.

\section{Proposition 3.2.}

$\left(\frac{\partial}{\partial t}-\Delta\right)\left(\frac{|\operatorname{Ric}|^{2}}{S^{2}}\right)=-\frac{2}{S^{4}}\left|S \nabla_{p} R_{i j}-\nabla_{p} S R_{i j}\right|^{2}-\frac{P}{S^{3}}+\left\langle\nabla\left(\frac{|\operatorname{Ric}|^{2}}{S^{2}}\right), \nabla \log S^{2}\right\rangle$,

where

$$
P=\frac{1}{2}\left((\mu+\nu-\lambda)^{2}(\mu-\nu)^{2}+(\lambda+\nu-\mu)^{2}(\lambda-\nu)^{2}+(\lambda+\mu-\nu)^{2}(\lambda-\mu)^{2}\right)
$$

with $\mu, \nu$ and $\lambda$ are eigenvalues of Ric. 
If $\left(M^{3}, g\right)$ is a gradient shrinking soliton, combining the discussion above we find that at $t=0$,

$$
\begin{aligned}
0= & \Delta\left(\frac{|\operatorname{Ric}|^{2}}{S^{2}}\right)-\left\langle\nabla\left(\frac{|\operatorname{Ric}|^{2}}{S^{2}}\right), \nabla f\right\rangle-\frac{2}{S^{4}}\left|S \nabla_{p} R_{i j}-\nabla_{p} S R_{i j}\right|^{2} \\
& -\frac{P}{S^{3}}+\left\langle\nabla\left(\frac{|\operatorname{Ric}|^{2}}{S^{2}}\right), \nabla \log S^{2}\right\rangle .
\end{aligned}
$$

Now multiply both sides of the above equation by $\mid$ Ric $\left.\right|^{2} e^{-f}$ and then integrate by parts. For the rest of the argument we will assume that all integrals involved are finite and the integration by parts can be performed, which we shall justify after we complete the (formal) argument.

$$
\begin{aligned}
0= & \int_{M}-\left\langle\nabla\left(\frac{|\operatorname{Ric}|^{2}}{S^{2}}\right), \nabla|\operatorname{Ric}|^{2}\right\rangle e^{-f}-\frac{2|\operatorname{Ric}|^{2}}{S^{4}}\left|S \nabla_{p} R_{i j}-\nabla_{p} S R_{i j}\right|^{2} e^{-f} \\
& \int_{M}-\frac{P}{S^{3}}|\operatorname{Ric}|^{2} e^{-f}+\left\langle\nabla\left(\frac{|\operatorname{Ric}|^{2}}{S^{2}}\right), \nabla \log S^{2}\right\rangle|\operatorname{Ric}|^{2} e^{-f}
\end{aligned}
$$

Since

we deduce that

$$
\nabla\left(\frac{|\mathrm{Ric}|^{2}}{S^{2}}\right)=\frac{\nabla|\operatorname{Ric}|^{2}}{S^{2}}-\frac{\nabla S^{2}}{S^{4}}|\operatorname{Ric}|^{2}
$$

$$
\begin{aligned}
& \int_{M}-\left\langle\nabla\left(\frac{|\operatorname{Ric}|^{2}}{S^{2}}\right), \nabla|\operatorname{Ric}|^{2}\right\rangle e^{-f}+\left\langle\nabla\left(\frac{|\operatorname{Ric}|^{2}}{S^{2}}\right), \nabla \log S^{2}\right\rangle|\operatorname{Ric}|^{2} e^{-f} \\
= & -\int_{M}\left|\nabla\left(\frac{|\operatorname{Ric}|^{2}}{S^{2}}\right)\right|^{2} S^{2} e^{-f} .
\end{aligned}
$$

Hence, we have that

$$
\begin{aligned}
0= & \int_{M}-\left|\nabla\left(\frac{|\operatorname{Ric}|^{2}}{S^{2}}\right)\right|^{2} S^{2} e^{-f}-\frac{2|\operatorname{Ric}|^{2}}{S^{4}}\left|S \nabla_{p} R_{i j}-\nabla_{p} S R_{i j}\right|^{2} e^{-f} \\
& \int_{M}-\frac{P}{S^{3}}|\operatorname{Ric}|^{2} e^{-f}
\end{aligned}
$$

In particular, $\frac{|\mathrm{Ric}|^{2}}{S^{2}}$ is a constant,

$$
S \nabla_{p} R_{i j}-\nabla_{p} S R_{i j}=0
$$

and $P=0$. If we choose a orthornormal frame such that $R_{i j}$ is diagonal, the equality (3.4) implies that

$$
\begin{aligned}
S \nabla_{p} R_{j j} & =\nabla_{p} S R_{j j} \\
S \nabla_{p} R_{i j} & =0, \text { for } i \neq j .
\end{aligned}
$$

Combining this with the second Bianchi identity we have

$$
\frac{1}{2} \nabla_{i} S=\sum_{p} \nabla_{p} R_{i p}=\nabla_{i} R_{i i}
$$

and

$$
\frac{1}{2} S \nabla_{i} S=S \nabla_{j} R_{j j}=\nabla_{i} S R_{i i}
$$


On the other hand, $P=0$ implies that $R_{11}=R_{22}=R_{33}=\frac{1}{3} S$. It follows that

$$
\frac{1}{2} S \nabla_{i} S=\left(\nabla_{i} S\right) \frac{S}{3}
$$

Hence $S$ is a constant. Now (3.5) and (3.6) imply that $\nabla_{p} R_{i j}=0$ for any $p, i, j$. This implies that $M$ is a compact locally symmetric space with positive curvature. The claim then follows from well known results.

Now with the help of Proposition 2.2 and Lemma 2.3 we now justify the finiteness of the integrals involved and the integration by parts.

First note that if we assume that $\sup _{x \in M}\left|R_{i j k l}\right|(x) \leq C$ for some $C>0$, namely the curvature is bounded, invoking the Bernstein-Bando-Shi type derivative estimates (cf. [CK], Theorem 7.1), we have that $\left|\nabla^{m} R_{i j k l}\right|$ are uniformly bounded on $M$. Hence all the integrals involved are finite which then implies, via cut-off function argument, that the integrations by parts are completely legal, in view of the fast decay of $e^{-f}$ ensured by Lemma 2.3 and the lower bound of $S$ provided by Proposition 2.2.

For the more general case that Ric satisfies (3.1), notice first that for three manifolds the assumption on $\mid$ Ric $\mid$ is equivalent to the same assumption on $\left|R_{i j k l}\right|$ (with a factor of absolute constant). Hence we have that for any $\epsilon>0$, there exists $\beta(\epsilon)>0$ such that

$$
\left|R_{i j k l}\right|(y, t) \leq \beta(r(x)+1)^{2}
$$

for all $y \in B_{g\left(-\frac{1}{2}\right)}\left(x, \frac{r(x)}{2}\right)$ and $t \in\left[-\frac{1}{2}, 0\right]$.

We estimate $\Delta\left(\frac{\mid \text { Ric }\left.\right|^{2}}{S^{2}}\right) \mid$ Ric $\left.\right|^{2}$. The other estimates are similar. Applying the local derivative estimate of Shi (cf. Theorem 13.1 of [H3]) we have that

$$
\begin{aligned}
\left|\nabla_{p} R_{i j k l}\right|(x, 0) & \leq C_{1}(r(x)+1)^{3} \\
\left|\nabla_{p} \nabla_{q} R_{i j k l}\right|(x, 0) & \leq C_{2}(r(x)+1)^{4} .
\end{aligned}
$$

A direct computation shows that

$$
\begin{aligned}
\Delta\left(\frac{|\operatorname{Ric}|^{2}}{S^{2}}\right)= & \frac{\Delta|\operatorname{Ric}|^{2}}{S^{2}}-2 \frac{\left\langle\nabla|\operatorname{Ric}|^{2}, \nabla \log S^{2}\right\rangle}{S^{2}}+2|\operatorname{Ric}|^{2} \frac{\left|\nabla \log S^{2}\right|^{2}}{S^{2}} \\
& -\frac{\Delta S^{2}}{S^{4}}|\operatorname{Ric}|^{2} .
\end{aligned}
$$

At $t=0$ there exist absolute constants $C_{i}, i=3,4,5$ and $\beta_{3}(\epsilon)$ depending only on $\beta_{1}$ and $\beta_{2}$ such that for $r(x)>>1$,

$$
\begin{aligned}
I= & \left(\left|\frac{\Delta|\operatorname{Ric}|^{2}}{S^{2}}\right||\operatorname{Ric}|^{2}\right)(x, 0) \leq C_{3}(r(x)+1)^{8} \\
I I= & \left(\left|\frac{\left\langle\nabla|\operatorname{Ric}|^{2}, \nabla \log S^{2}\right\rangle}{S^{2}}\right||\operatorname{Ric}|^{2}+|\operatorname{Ric}|^{4} \frac{\left|\nabla \log S^{2}\right|^{2}}{S^{2}}\right)(x, 0) \\
& \leq C_{4}(r(x)+1)^{14}, \\
I I I= & \left(\left|\frac{\Delta S^{2}}{S^{4}}\right||\operatorname{Ric}|^{4}\right)(x, 0) \leq C_{5}(r(x)+1)^{14}
\end{aligned}
$$


Putting the above estimates together with (2.4) we conclude that if $t=0$ then

$$
\int_{M}\left|\Delta\left(\frac{|\operatorname{Ric}|^{2}}{S^{2}}\right)\right||\operatorname{Ric}|^{2} e^{-f} d \mu_{0}<\infty .
$$

Similarly, one can establish the finiteness of the other integrals involved. Once we have the the finiteness of the integration, the integration by parts can be checked by approximation via the cut-off functions. This is somewhat standard we hence leave the details to the reader.

Remark 3.3. A similar argument was used by Huisken [Hu2] in the classification of mean convex shrinking solitons of mean curvature flow in $\mathbb{R}^{n+1}$.

\section{Preliminaries for higher dimensions}

Most of the results in this section are either known (cf. [Hu1, H2]) or can be derived easily from the literature. We include them here for completeness and in order to adapt the statements to our specific needs. We recall the Ricci flow evolution formula for the curvature [H1]:

$$
\begin{aligned}
\left(\frac{\partial}{\partial t}-\Delta\right) R_{i j k l}= & 2\left(\mathbf{R}^{2}+\mathbf{R}^{\#}\right)_{i j k l} \\
& -\left(R_{i p} R_{p j k l}+R_{j p} R_{i p k l}+R_{k p} R_{i j p l}+R_{l p} R_{i j k p}\right)
\end{aligned}
$$

where $Q(\mathbf{R})=\mathbf{R}^{2}+\mathbf{R}^{\#}$ is defined via the Lie algebra structure of $\wedge^{2}(n)$, which can be identified with the Lie algebra of $O(n)$. The below is a brief account.

Let $(E, g)$ be a Euclidean space with metric $g$. We can make the following identifications: $\otimes^{2} E$, the tensor space, can be identified with $G L(n, \mathbb{R})$, the linear transformations on $E$ (for any $x \otimes y \in \otimes^{2} E, x \otimes y(z)=\langle y, z\rangle x$ is the corresponding element of $M_{n}(\mathbb{R})$ ); under this identification, the space symmetric two tensors $S^{2} E$ corresponds to the symmetric transformations $S^{2}(E) ; \wedge^{2} E$ can be identified with $s o(n)$ $\left(e_{i} \wedge e_{j}=e_{i} \otimes e_{j}-e_{j} \otimes e_{i}\right.$ is identified with the matrix $e_{i j}$ with 1 at $(i, j)$-th position and -1 at $(j, i)$-th position and zeros everywhere else. The metric on $T M$ extends naturally to all the related tensor spaces such as $\otimes^{2} T M, S^{2} T M, \wedge^{2} T M$. With respect to the previous identification, the metric on $s o(n)$ is given by $\langle A, B\rangle=-\frac{1}{2} \operatorname{tr}(A B)$ $\left(=\frac{1}{2} \operatorname{tr}\left(A^{t} B\right)\right)$ thus $\left\{e_{i} \wedge e_{j}\right\}_{i<j}$ is an orthonormal basis of $\left.\wedge^{2} T M\right)$. The identification also equips $\wedge^{2} T M$ with a Lie algebra structure, which is of fundamental importance in the study of evolution of curvature operators under Ricci flow. This was first observed by Hamilton [H2]. Let us recall this fact first. For an orthonormal basis $\phi_{\alpha}$ of $\wedge^{2} T M$ (say $\phi_{\alpha}=e_{i} \wedge e_{j}$, which is identified with $e_{i j}$ ), the Lie bracket is given by

$$
\left[\phi_{\alpha}, \phi_{\beta}\right]=c_{\alpha \beta \gamma} \phi_{\gamma}
$$

It is easy to check, by simple linear algebra, that

$$
\langle[\phi, \psi], \omega\rangle=-\langle[\omega, \psi], \phi\rangle .
$$

This immediately implies that $c_{\alpha \beta \gamma}$ is anti-symmetric. If $A, B \in S^{2}\left(\wedge^{2} T M\right)$ one can define

$$
(A \# B)_{\alpha \beta}=\frac{1}{2} c_{\alpha \gamma \eta} c_{\beta \delta \theta} A_{\gamma \delta} B_{\eta \theta} \text {. }
$$

It is easy to see that $A \# B$ is symmetric too. Also from the anti-symmetry of $c_{\alpha \beta \gamma}$

$$
A \# B=B \# A \text {. }
$$


The easy computation also shows that

$$
\langle(A \# B)(\phi), \psi\rangle=\frac{1}{2} \sum_{\alpha \beta}\left\langle\left[A\left(\omega_{\alpha}\right), B\left(\omega_{\beta}\right)\right], \phi\right\rangle \cdot\left\langle\left[\omega_{\alpha}, \omega_{\beta}\right], \psi\right\rangle
$$

if $\left\{\omega_{\alpha}\right\}$ is an orthonormal basis. This particularly implies that $\operatorname{tr}((A \# B) \cdot C)$ is symmetric in $A, B, C$ since

$$
\begin{aligned}
\operatorname{tr}((A \# B) \cdot C) & =\sum_{\gamma}\left\langle(A \# B) \cdot C\left(\omega_{\gamma}\right), \omega_{\gamma}\right\rangle \\
& =\frac{1}{2} \sum_{\alpha \beta \gamma}\left\langle\left[A\left(\omega_{\alpha}\right), B\left(\omega_{\beta}\right)\right], C\left(\omega_{\gamma}\right)\right\rangle\left\langle\left[\omega_{\alpha}, \omega_{\beta}\right], \omega_{\gamma}\right\rangle .
\end{aligned}
$$

Now define

$$
\operatorname{tri}(A, B, C)=\operatorname{tr}((A B+B A+2 A \# B) C)
$$

which is symmetric in all three variables. If we write

$$
\mathbf{R}\left(e_{i} \wedge e_{j}\right)=\frac{1}{2} \sum_{k, l} R_{i j k l} e_{k} \wedge e_{l}
$$

we would have that

$$
\left|R_{i j k l}\right|^{2}=4\langle\mathbf{R}, \mathbf{R}\rangle .
$$

We denote $\operatorname{tri}(\mathbf{R})=\operatorname{tri}(\mathbf{R}, \mathbf{R}, \mathbf{R})=\left\langle 2\left(\mathbf{R}^{2}+\mathbf{R}^{\#}\right), \mathbf{R}\right\rangle$ and $Q(\mathbf{R})=\mathbf{R}^{2}+\mathbf{R}^{\#}$.

The curvature operator $\mathbf{R}$ has an orthogonal splitting, with respect irreducible $O(n)$ representation, into the trace part $\mathbf{R}_{\mathrm{I}}=\frac{S}{n(n-1)} \mathrm{I}$, the traceless Ricci part $\mathbf{R}_{\mathrm{Ric}_{0}}=$ $\frac{2}{n-2} \operatorname{Ric}_{0} \wedge \mathrm{id}$, where $\mathrm{Ric}_{0}$ denotes the traceless part of the Ricci curvature, and the Weyl curvature $\mathbf{R}_{W}$ (cf. [BW1]). We denote the three subspaces by $\langle\mathrm{I}\rangle,\left\langle\mathrm{Ric}_{0}\right\rangle$ and $\langle W\rangle$ respectively. Equipped with the above notation we have that

Lemma 4.1.

$$
\left(\frac{\partial}{\partial t}-\Delta\right)\left|R_{i j k l}\right|^{2}=8 \operatorname{tri}(\mathbf{R})-2\left|\nabla_{p} R_{i j k l}\right|^{2}
$$

The following result is a direct consequence.

Proposition 4.2. Assume that $S \neq 0$. Then

$$
\begin{aligned}
& \left(\frac{\partial}{\partial t}-\Delta\right)\left(\frac{\left|R_{i j k l}\right|^{2}}{S^{2}}\right)=\frac{4}{S^{3}}\left(2 \operatorname{tri}(\mathbf{R}) S-\sigma^{2}\left|R_{i j k l}\right|^{2}\right) \\
& -\frac{2}{S^{4}}\left|S \nabla_{p} R_{i j k l}-\nabla_{p} S R_{i j k l}\right|^{2}+\left\langle\nabla\left(\frac{\left|R_{i j k l}\right|^{2}}{S^{2}}\right), \nabla \log S^{2}\right\rangle,
\end{aligned}
$$

where $\sigma^{2}=\mid$ Ric $\left.\right|^{2}$.

Recall that Tachibana [T] proved that (see also [CLN], pages 267-269), under the assumption that $\mathbf{R} \geq 0$,

$$
-2 \operatorname{tri}(\mathbf{R})+\operatorname{Ric}(\mathbf{R}, \mathbf{R}) \geq 0
$$

where $\operatorname{Ric}(\mathbf{R}, \mathbf{R})=R_{i p} R_{i j k l} R_{p j k l}$. 
In [Hu1], Huisken obtained the following identities.

$$
\begin{aligned}
& \left(\mathbf{R}_{\mathrm{I}}\right)_{i j k l}(Q(\mathbf{R}))_{i j k l}=4\left\langle Q(\mathbf{R}), \mathbf{R}_{\mathrm{I}}\right\rangle=\frac{2}{n(n-1)} S \sigma^{2} \\
& \left(\mathbf{R}_{\mathrm{Ric}_{0}}\right)_{i j k l}(Q(\mathbf{R}))_{i j k l}=\frac{4}{n(n-1)} S \tilde{\sigma}^{2}-\frac{8}{(n-2)^{2}} \lambda_{i}^{3}+\frac{4}{n-2}\left(\mathbf{R}_{W}\right)_{i j i j} \lambda_{i} \lambda_{j} \\
& \left(\mathbf{R}_{W}\right)_{i j k l}(Q(\mathbf{R}))_{i j k l}=2 \operatorname{tri}\left(\mathbf{R}_{W}\right)+\frac{2}{n-2}\left(\mathbf{R}_{W}\right)_{i j i j} \lambda_{i} \lambda_{j}
\end{aligned}
$$

where $\lambda_{i}$ are the eigenvalues of $\operatorname{Ric}_{0}$ and $\tilde{\sigma}^{2}=\sum \lambda_{i}^{2}$. Below we first show these equations via the following lemma, which essentially follows from [BW1]. In [Hu1], the result was shown by direct but long computations which were omitted. With the help of [BW1], the result can be obtained without much computation. We include the derivation for the sake of completeness. First we need the following lemma essentially proved in $[\mathrm{BW} 1]$.

\section{Lemma 4.3.}

$$
\mathbf{R}+\mathbf{R} \# \mathrm{I}=\operatorname{Ric}(\mathbf{R}) \wedge \text { id } .
$$

Hence for any $\mathbf{R}_{1}, \mathbf{R}_{2} \in S_{B}\left(\wedge^{2}(n)\right)$, let

$$
B\left(\mathbf{R}_{1}, \mathbf{R}_{2}\right)=\mathbf{R}_{1} \mathbf{R}_{2}+\mathbf{R}_{2} \mathbf{R}_{1}+2 \mathbf{R}_{1} \# \mathbf{R}_{2} .
$$

Let $\mathbf{R}_{I}^{i} \in\langle\mathrm{I}\rangle, \mathbf{R}_{0} \in\left\langle\operatorname{Ric}_{0}\right\rangle, W_{i}, W \in\langle W\rangle(i=1,2)$. Then the following hold

$$
\begin{aligned}
B\left(\mathbf{R}_{\mathrm{I}}, W\right) & =0 \\
B\left(\mathbf{R}_{\mathrm{I}}^{1}, \mathbf{R}_{\mathrm{I}}^{2}\right) & \in\langle\mathrm{I}\rangle \\
B\left(W_{1}, W_{2}\right) & \in\langle W\rangle \\
B\left(\mathbf{R}_{\mathrm{I}}, \mathbf{R}_{0}\right) & \in\left\langle\operatorname{Ric}_{0}\right\rangle \\
B\left(\mathbf{R}_{0}, W\right) & \in\left\langle\operatorname{Ric}_{0}\right\rangle \\
\frac{1}{2} B\left(\mathbf{R}_{0}, \mathbf{R}_{0}\right) & =\frac{1}{n-2} \operatorname{Ric}_{0} \wedge \operatorname{Ric}_{0}-\frac{2}{(n-2)^{2}}\left(\operatorname{Ric}_{0}^{2}\right)_{0} \wedge \mathrm{id}+\frac{\tilde{\sigma}^{2}}{n(n-2)} \mathrm{I} .
\end{aligned}
$$

Moreover

$$
\operatorname{Ric}_{0} \wedge \operatorname{Ric}_{0}=-\frac{\tilde{\sigma}^{2}}{n(n-1)} \mathrm{I}-\frac{2}{n-2}\left(\operatorname{Ric}_{0}^{2}\right)_{0} \wedge \mathrm{id}+\left(\operatorname{Ric}_{0} \wedge \operatorname{Ric}_{0}\right)_{W} .
$$

The above lemma will be used in the following calculations.

$$
\begin{aligned}
\operatorname{tri}\left(\mathbf{R}, \mathbf{R}, \mathbf{R}_{\mathrm{I}}\right) & =\operatorname{tri}\left(\mathbf{R}, \mathbf{R}_{\mathrm{I}}, \mathbf{R}\right) \\
& =\frac{2 S}{n(n-1)}\langle\operatorname{Ric} \wedge \mathrm{id}, \mathbf{R}\rangle \\
& =\frac{2 S}{n(n-1)}\left\langle\frac{S}{n} \mathrm{id} \wedge \mathrm{id}+\operatorname{Ric}_{0} \wedge \mathrm{id}, \frac{S}{n(n-1)} \mathrm{id} \wedge \mathrm{id}+\frac{2}{n-2} \operatorname{Ric}_{0} \wedge \mathrm{id}\right\rangle
\end{aligned}
$$


Set $\bar{\lambda}=\frac{S}{n}$. Then

$$
\begin{aligned}
& \left\langle\frac{S}{n} \mathrm{id} \wedge \mathrm{id}+\operatorname{Ric}_{0} \wedge \mathrm{id}, \frac{S}{n(n-1)} \mathrm{id} \wedge \mathrm{id}+\frac{2}{n-2} \operatorname{Ric}_{0} \wedge \mathrm{id}\right\rangle \\
= & \left\langle\bar{\lambda} \mathrm{id} \wedge \mathrm{id}+\operatorname{Ric}_{0} \wedge \mathrm{id}, \frac{\bar{\lambda}}{n-1} \mathrm{id} \wedge \mathrm{id}+\frac{2}{n-2} \operatorname{Ric}_{0} \wedge \mathrm{id}\right\rangle=\frac{n}{2} \bar{\lambda}^{2}+\frac{1}{2} \sum \lambda_{i}^{2} \\
= & \frac{1}{2} \sigma^{2} .
\end{aligned}
$$

This proves (4.3). For (4.4), let $\mathbf{R}_{0}=\mathbf{R}_{\mathrm{Ric}_{0}}$. We next compute $\operatorname{tri}\left(\mathbf{R}, \mathbf{R}, \mathbf{R}_{0}\right)$. Using the symmetry

$$
\begin{aligned}
\operatorname{tri}\left(\mathbf{R}, \mathbf{R}, \mathbf{R}_{0}\right) & =\operatorname{tri}\left(\mathbf{R}, \mathbf{R}_{0}, \mathbf{R}\right) \\
& =\left\langle B\left(\mathbf{R}_{\mathrm{I}}, \mathbf{R}_{0}\right), \mathbf{R}\right\rangle+\left\langle B\left(\mathbf{R}_{0}, \mathbf{R}_{0}\right), \mathbf{R}\right\rangle+\left\langle B\left(\mathbf{R}_{W}, \mathbf{R}_{0}\right), \mathbf{R}\right\rangle \\
& =\left\langle B\left(\mathbf{R}_{\mathrm{I}}, \mathbf{R}_{0}\right), \mathbf{R}_{0}\right\rangle+\left\langle B\left(\mathbf{R}_{0}, \mathbf{R}_{0}\right), \mathbf{R}_{\mathrm{I}}+\mathbf{R}_{0}+\mathbf{R}_{W}\right\rangle+\left\langle B\left(\mathbf{R}_{W}, \mathbf{R}_{0}\right), \mathbf{R}_{0}\right\rangle \\
& =2 \operatorname{tri}\left(\mathbf{R}_{0}, \mathbf{R}_{0}, \mathbf{R}_{\mathrm{I}}\right)+2 \operatorname{tri}\left(\mathbf{R}_{0}, \mathbf{R}_{0}, \mathbf{R}_{W}\right)+\operatorname{tri}\left(\mathbf{R}_{0}, \mathbf{R}_{0}, \mathbf{R}_{0}\right) .
\end{aligned}
$$

Using (4.7) and (4.8) we have that

$$
\operatorname{tri}\left(\mathbf{R}_{0}, \mathbf{R}_{0}, \mathbf{R}_{\mathrm{I}}\right)=\frac{1}{n(n-1)} \tilde{\sigma}^{2} S
$$

Similarly,

and

$$
\operatorname{tri}\left(\mathbf{R}_{0}, \mathbf{R}_{0}, \mathbf{R}_{0}\right)=-\frac{4}{(n-2)^{2}} \sum \lambda_{i}^{3}
$$

$$
2 \operatorname{tri}\left(\mathbf{R}_{0}, \mathbf{R}_{0}, \mathbf{R}_{W}\right)=\frac{2}{n-2}\left(R_{W}\right)_{i j i j} \lambda_{i} \lambda_{j} .
$$

The above three equations give (4.4). For (4.5), notice that

$$
\operatorname{tri}\left(\mathbf{R}_{W}, \mathbf{R}, \mathbf{R}\right)=\operatorname{tri}\left(\mathbf{R}_{0}, \mathbf{R}_{W}, \mathbf{R}_{0}\right)+\operatorname{tri}\left(\mathbf{R}_{W}, \mathbf{R}_{W}, \mathbf{R}_{W}\right) .
$$

Then the claimed equality follows from the above computation on $\operatorname{tri}\left(\mathbf{R}_{0}, \mathbf{R}_{0}, \mathbf{R}_{W}\right)$.

We have, finally, arrived at the following formula:

$$
\begin{aligned}
2 \operatorname{tri}(\mathbf{R}) S-\sigma^{2}\left|R_{i j k l}\right|^{2}= & -4\left|\mathbf{R}_{W}\right|^{2} \sigma^{2}+2 S \operatorname{tri}\left(\mathbf{R}_{W}, \mathbf{R}_{W}, \mathbf{R}_{W}\right) \\
& -\frac{4}{n(n-1)(n-2)} S^{2} \tilde{\sigma}^{2}-\frac{4}{n-2} \tilde{\sigma}^{4} \\
& -\frac{8}{(n-2)^{2}} S \sum \lambda_{i}^{3}+\frac{6}{n-2} S\left(\mathbf{R}_{W}\right)_{i j i j} \lambda_{i} \lambda_{j} .
\end{aligned}
$$

This follows from (4.3)-(4.5) along with the observation that

$$
\begin{aligned}
|\mathbf{R}|^{2} & =\left|\mathbf{R}_{\mathrm{I}}\right|^{2}+\left|\mathbf{R}_{\text {Ric }_{0}}\right|^{2}+\left|\mathbf{R}_{W}\right|^{2} \\
& =\frac{S^{2}}{2 n(n-1)}+\frac{1}{n-2} \sum \lambda_{j}^{2}+\left|\mathbf{R}_{W}\right|^{2}
\end{aligned}
$$

and

Hence

$$
\sigma^{2}=\frac{S^{2}}{n}+\tilde{\sigma}^{2}
$$

$$
4 \sigma^{2}|\mathbf{R}|^{2}=\frac{2 S^{2}}{n(n-1)} \sigma^{2}+\frac{4 S^{2} \tilde{\sigma}^{2}}{(n-2) n}+\frac{4}{n-2} \tilde{\sigma}^{4}+4\left|\mathbf{R}_{W}\right|^{2} \sigma^{2}
$$


In the case that $\mathbf{R}_{W}=0$, which is automatical if $n=3$ and amounts to that $(M, g)$ is locally conformally flat if $n \geq 4$, we have that

$$
2 \operatorname{tri}(\mathbf{R}) S-\sigma^{2}\left|R_{i j k l}\right|^{2}=-\frac{4}{n(n-1)(n-2)} S^{2} \tilde{\sigma}^{2}-\frac{4}{n-2} \tilde{\sigma}^{4}-\frac{8}{(n-2)^{2}} S \sum \lambda_{i}^{3}
$$

Similarly, since

$$
\left(\frac{\partial}{\partial t}-\Delta\right) R_{i k}=2 R_{i j k l} R_{j l}-2 R_{i l} R_{l k}
$$

we also have the following high dimensional analogue of Proposition 3.2.

Proposition 4.4. Assume that $S>0$. Then

$$
\begin{aligned}
& \left(\frac{\partial}{\partial t}-\Delta\right)\left(\frac{\sigma^{2}}{S^{2}}\right)=\frac{4}{S^{3}}\left(S R_{i j k l} R_{j l} R_{i k}-\sigma^{4}\right) \\
& -\frac{2}{S^{4}}\left|S \nabla_{p} R_{i j}-\nabla_{p} S R_{i j}\right|^{2}+\left\langle\nabla\left(\frac{\sigma^{2}}{S^{2}}\right), \nabla \log S^{2}\right\rangle .
\end{aligned}
$$

In the case $\operatorname{dim}(M)=3$ the above implies Hamilton's computation (Proposition $3.2)$

\section{High dimensional locally conformally flat manifolds}

We first prove the following algebraic result.

Proposition 5.1. Let $\sigma, \tilde{\sigma}, \lambda_{i}$ be as in the last section. Then

$$
-\frac{4}{n-2}\left(\frac{1}{n(n-1)} S^{2} \tilde{\sigma}^{2}+\tilde{\sigma}^{4}+\frac{2}{n-2} S \sum \lambda_{i}^{3}\right) \leq 0 .
$$

If the equality holds, then either

(i) $\lambda_{i}=0$ for all $1 \leq i \leq n$, or

(ii) there exists $a>0$ such that

$$
\begin{aligned}
\lambda_{l} & =\frac{1}{\sqrt{n(n-1)}} a, \quad \text { for } 1 \leq l \leq n-1 ; \\
\lambda_{n} & =-\sqrt{\frac{n-1}{n}} a
\end{aligned}
$$

and $S=\sqrt{n(n-1)}$ a.

Proof. Let

$$
F\left(S, \lambda_{1}, \cdots, \lambda_{n}\right)=\frac{1}{n(n-1)} S^{2} \sum \lambda_{i}^{2}+\frac{2}{n-2} S \sum \lambda_{i}^{3}+\left(\sum \lambda_{i}^{2}\right)^{2} .
$$

The goal is to show that $F \geq 0$ under the constraint that $\sum \lambda_{i}=0$ and analyze the equality case. Since it is homogenous we can consider the extremal values of $F$ under the further constraint $\sum \lambda_{i}^{2}=1$. Viewing $F$ as a quadratic form in terms of $S$, the result follows, by elementary consideration, if we show that

$$
\left(\sum \lambda_{i}^{3}\right)^{2} \leq \frac{(n-2)^{2}}{n(n-1)}
$$


under the constraints $\sum \lambda_{i}=0$ and $\sum \lambda_{i}^{2}=1$. Let $G=\sum_{i} \lambda_{i}^{3}$. By the method of Lagrange multipliers we find the following identities at the critical points.

$$
\begin{aligned}
3 \lambda_{j}^{2}-\lambda-2 \mu \lambda_{j} & =0, \quad \text { for } 1 \leq j \leq n, \\
\sum \lambda_{i} & =0 \\
\sum \lambda_{i}^{2} & =1 .
\end{aligned}
$$

This implies that $\lambda=\frac{3}{n}$ and

$$
\lambda_{j}=\frac{\mu+\epsilon_{j} \sqrt{\mu^{2}+\frac{9}{n}}}{3}
$$

with $\epsilon_{j} \in\{-1,1\}$. We shall compute all possible values of $\lambda_{j}$. The problem splits into two cases.

Case 1: $n=2 k$. Let $\epsilon=\sum_{j} \epsilon_{j}$ which takes value in $\{-2 k,-2(k-1), \cdots,-2,0,2, \cdot \cdot$ $\cdot, 2(k-1), 2 k\}$. Since $\sum_{j} \lambda_{j}=0$, it is easy to see that $\epsilon$ can not take the value $2 k$ or $-2 k$. If $\epsilon=0$ then $\mu=0$, which then implies, after a permutation of the indices, that $\lambda_{j}=\frac{1}{\sqrt{n}}$ for $1 \leq j \leq k$ and $\lambda_{j}=-\frac{1}{\sqrt{n}}$ for $k \leq j \leq 2 k$. In this case $G=0$.

In general assume that $\epsilon=2(k-i)$ for some $1 \leq i \leq k$. We shall consider only the range $1 \leq i \leq k-1$ since the other values of $i$ follow by symmetry. Without the loss of the generality we may assume that $\epsilon_{j}=1$ for $1 \leq j \leq 2 k-i$ and $\epsilon_{j}=-1$ for $2 k-i \leq j \leq 2 k$. In this case

$$
\begin{aligned}
\mu & =-\frac{3(k-i)}{\sqrt{(2 k-i) 2 k i}}, \\
\lambda_{l} & =\sqrt{\frac{i}{2 k(2 k-i)}}, \quad \text { if } 1 \leq l \leq 2 k-i, \\
\lambda_{l} & =-\sqrt{\frac{2 k-i}{2 k i}}, \quad \text { if } 2 k-i<l \leq 2 k .
\end{aligned}
$$

This implies that

$$
G=-\frac{n-2 i}{\sqrt{(n-i) i} \sqrt{n}} .
$$

Since $\frac{(n-2 i)^{2}}{(n-i) i}$ is monotone decreasing in $i$, we can conclude that $G \geq-\frac{n-2}{\sqrt{n(n-1)}}$. Symmetrically, for $\epsilon=-2(k-i)$ we can find $G=\frac{n-2 i}{\sqrt{(n-i) i} \sqrt{n}}$. Combining these observations we have

$$
-\frac{n-2}{\sqrt{n(n-1)}} \leq G \leq \frac{n-2}{\sqrt{n(n-1)}} .
$$

The minimum is achieved when $i=1$, which implies the second part of the statement in the proposition in this case.

Case 2: $n=2 k+1$. Again due to the fact that $\sum \lambda_{i}=0, \epsilon$ takes values in $\{-(2 k-1), \cdots,-1,1, \cdots, 2 k-1\}$. Assume that $\epsilon=2(k-i)+1$ for some $1 \leq i \leq 2 k$. As above we shall invoke symmetry and only consider $1 \leq i \leq k$. Now we assume 
that $\epsilon_{j}=1$ for all $1 \leq j \leq 2 k-i+1$, and $\epsilon_{j}=-1$ for $2 k-i+2 \leq j \leq 2 k+1$. Now we have that

$$
\begin{aligned}
\mu & =-\frac{3}{2} \cdot \frac{2(k-i)+1}{\sqrt{2 k+1} \sqrt{i} \sqrt{2 k-i+1}} \\
\lambda_{l} & =\frac{\sqrt{j}}{\sqrt{2 k-j+1} \sqrt{2 k+1}}, \quad \text { for } \quad 1 \leq 1 \leq l \leq 2 k-i+1, \\
\lambda_{l} & =-\frac{\sqrt{2 k-i+1}}{\sqrt{i} \sqrt{2 k+1}}, \quad \text { for } \quad 2 k-i+2 \leq l \leq 2 k+1
\end{aligned}
$$

From this we can compute that

$$
G=-\frac{n-2 i}{\sqrt{n} \sqrt{n-i} \sqrt{i}}
$$

Since

$$
-\frac{n-2 i}{\sqrt{n-i} \sqrt{i}} \geq-\frac{n-2}{\sqrt{n-1} \sqrt{n}}
$$

we conclude that $G^{2} \leq \frac{(n-2)^{2}}{(n-1) n}$. The minimum is therefore achieved when $i=1$.

Combining the above two cases, we complete the proof that $F \geq 0$. From the above discussion, it is straight forward to check that the listed cases are the only two that can achieve the equality.

Corollary 5.2. Let $\left(M^{n}, g\right)(n \geq 4)$ be a locally conformally flat gradient shrinking soliton whose Ricci curvature is nonnegative. Then either $M$ is isometric to $\mathbb{R}^{n}$, or it is a quotient of $\mathbb{S}^{n}$ or $\mathbb{S}^{n-1} \times \mathbb{R}$. In the case that $M$ is compact, the assumption that the Ricci curvature is nonnegative is not needed.

In particular, if $\left(M^{n}, g\right)$ has positive Ricci curvature it must be compact.

Proof. Notice that $S$ satisfies the equation $\left(\frac{\partial}{\partial t}-\Delta\right) S=2|\operatorname{Ric}|^{2}$. If $M$ is compact Lemma 2.18 of [CLN] implies that $S \geq 0$. Hence in either the case $M$ is compact or the case $M$ is noncompact with Ric $\geq 0$, we all have that $S \geq 0$. By the strong maximum principle we know that if $S=0$ for some $\left(x_{0}, t_{0}\right)$, then $S(x, t) \equiv 0$. Therefore Ric $\equiv 0$ by the above parabolic equation. Using the soliton equation which now becomes $f_{i j}=\frac{1}{2} g_{i j}$, we conclude that $f=\frac{1}{2} r^{2}(x, o)$ where $o$ is the unique critical point of $f$. Now the Laplace comparison theorem implies that $M=\mathbb{R}^{n}$. Hence we may assume that $S>0$.

As in Section 3 we have that

$$
\begin{aligned}
0= & \Delta\left(\frac{\left|R_{i j k l}\right|^{2}}{S^{2}}\right)-\left\langle\nabla\left(\frac{\left|R_{i j k l}\right|^{2}}{S^{2}}\right), \nabla f\right\rangle-\frac{2}{S^{4}}\left|S \nabla_{p} R_{i j k l}-\nabla_{p} S R_{i j k l}\right|^{2} \\
& -\frac{P}{S^{3}}+\left\langle\nabla\left(\frac{\left|R_{i j k l}\right|^{2}}{S^{2}}\right), \nabla \log S^{2}\right\rangle .
\end{aligned}
$$

Here

$$
P=-4\left(2 \operatorname{tri}(\mathbf{R}) S-\sigma^{2}\left|R_{i j k l}\right|^{2}\right)
$$


which is nonnegative by Proposition 5.1. Multiplying $\left|R_{i j k l}\right|^{2} e^{-f}$ and integrating by parts, which can be justified similarly as in Section 3, we have that

$$
\begin{aligned}
0= & \int_{M}-\left|\nabla\left(\frac{\left|R_{i j k l}\right|^{2}}{S^{2}}\right)\right|^{2} S^{2} e^{-f}-\frac{2\left|R_{i j k l}\right|^{2}}{S^{4}}\left|S \nabla_{p} R_{i j k l}-\nabla_{p} S R_{i j k l}\right|^{2} e^{-f} \\
& \int_{M}-\frac{P}{S^{3}}\left|R_{i j k l}\right|^{2} e^{-f} .
\end{aligned}
$$

By Proposition 5.1 we have that

$$
\nabla_{p} S R_{i j k l}=S \nabla_{p} R_{i j k l}
$$

which implies that

$$
\nabla_{p} S R_{i k}=S \nabla_{p} R_{i k}
$$

Also the argument of Section 3 implies that

$$
2 \operatorname{tri}(\mathbf{R}) S-\sigma^{2}\left|R_{i j k l}\right|^{2}=-2\left(\frac{1}{12} S^{2} \tilde{\sigma}^{2}+\tilde{\sigma}^{4}+S \sum \lambda_{i}^{3}\right)=0
$$

and $\frac{\left|R_{i j k l}\right|^{2}}{S^{2}}$ is a constant.

If $\lambda_{i}=0$, then $R_{i k}=\frac{S}{n} \delta_{i k}$. The second Bianchi identity implies that

$$
\frac{1}{2} S \nabla_{i} S=S \nabla_{p} R_{i p}=\frac{S}{4} \delta_{i p} \nabla_{p} S .
$$

which implies that $\nabla_{p} S=0$. Then we have $\nabla_{p} R_{i j k l}=0$ by $(5.2)$.

If the second case of Proposition 5.1 happens, we have that $R_{i j}=\frac{\delta_{i j}}{n-1} S$ for $1 \leq$ $i, j \leq n-1$ and $R_{n j}=0$ for $1 \leq j \leq n$. The same computation as in $n=3$ shows that $\nabla_{p} S=0$, hence $\nabla_{p} R_{i j k l}=0$, which means that $(M, g)$ is locally symmetric. The conclusion follows from the fact that $(M, g)$ is either Einstein or its Ricci curvature has constant rank $n-1$ and with $n-1$ identical nonzero eigenvalues.

Remark 5.3. (1) The last assertion of the corollary should be compared with the result in $[\mathrm{NW}]$. In that paper, a certain curvature operator pinching condition is used to prove that the manifold is compact.

(2) Whether or not the argument here (or some variation of it) is sufficient to show that any shrinking gradient soliton with positive curvature operator must be compact is an interesting question. The Kähler case has been resolved in [N]. We hope to return to the remaining cases in the future study.

Since Proposition 5.1 also holds when $n=3$, and $\mathbf{R}_{W}=0$ automatically we have the following corollary which generalizes Theorem 1.1.

Corollary 5.4. Let $\left(M^{3}, g\right)$ be a gradient shrinking soliton whose Ricci curvature is nonnegative. Then either $M$ is isometric to $\mathbb{R}^{3}$, or it is a quotient of $\mathbb{S}^{3}$ or $\mathbb{S}^{2} \times \mathbb{R}$. In the case that $M$ is compact, the assumption on the nonnegativity of the Ricci curvature is not needed. In particular, if $\left(M^{3}, g\right)$ has positive Ricci curvature it must be compact.

Remark 5.5. After the submission of the current paper, in a recent preprint Petersen and Wylie observed that the nonnegativity of the Ricci curvature in Corollary 5.2 can be replaced by the assumption that $\mid$ Ric $\mid$ is bounded. One can also modify our 
argument above to prove this by doing integration over balls of radius $R$ and then taking $R \rightarrow \infty$.

\section{References}

[BW1] C. Böhm and B. Wilking, Manifolds with positive curvature operator are space form. To appear in Ann. of Math.

[BW2] C. Böhm and B. Wilking, New Ricci flow invariant curvature conditions in large dimension. Work in progress.

[CZ] H.-D. Cao and X.-P. Zhu, A complete proof of the Poincar and geometrization conjecturesapplication of the Hamilton-Perelman theory of the Ricci flow. Asian J. Math. 10 (2006), no. $2,165-492$.

[CK] B. Chow and D. Knopf, The Ricci flow: an introduction. Mathematical Surveys and Monographs, 110. American Mathematical Society, Providence, RI, 2004. xii+325 pp.

[CLN] B. Chow; P. Lu and L. Ni, Hamilton's Ricci flow. Graduate Studies in Mathematics, 77. American Mathematical Society, Providence, RI; Science Press, New York, 2006. xxxvi+608 pp.

[H1] R. Hamilton, Three-manifolds with positive Ricci curvature. J. Differential Geom. 17 (1982), no. 2, 255-306.

[H2] R. Hamilton, Four-manifolds with positive curvature operator. J. Differenital. Geom. 24 (1986 ), 153-179.

[H3] R. Hamilton, The formation of singularities in Ricci flow. Surveys in differential geometry, Vol. II (Cambridge, MA, 1993), 7-136, Int. Press, Cambridge, MA, 1995.

[Hu1] G. Huisken, Ricci deformation of metric on a Riemannian manifold. J. Differenital. Geom. 24 (1985), 47-62.

[Hu2] G. Huisken, Local and global behaviour of hypersurfaces moving by mean curvature. Differential geometry: partial differential equations on manifolds (Los Angeles, CA, 1990), 175-191, Proc. Sympos. Pure Math., 54, Part 1, Amer. Math. Soc., Providence, RI, 1993.

[I] T. Ivey, Ricci solitons on compact three-manifolds. Differential Geom. Appl. 3 (1993), no. 4, 301-307.

[K] B. Kotschwar, On rotationally invariant shrinking gradient Ricci solitons, Arxiv, math0702597.

[KL] B. Kleiner and J. Lott, Notes on Perelman's papers. ArXiv: math.DG/ 0605667.

[MT] J. Morgan and G. Tian, Ricci Flow and the Poincare Conjecture. ArXiv: math.DG/ 0607607.

[N] L. Ni, Ancient solutions to Kähler-Ricci flow. Math. Res. Lett. 12 (2005), no. 5-6, 633-653.

[NW] L. Ni and B. Wu, Complete manifolds with nonnegative curvature operator. Proceedings of AMS. 135 (2007), 3021-3028.

[P1] G. Perelman, The entropy formula for the Ricci flow and its geometric applications. ArXiv: math.DG/ 0211159 .

[P2] G. Perelman, Ricci flow with surgery on three-manifolds. ArXiv: math.DG/ 0303109.

[Sh] W. X. Shi, Deforming the metric on complete Riemannian manifolds. J. Differential Geom.30 (1989), 223-301.

[T] S. Tachibana, A theorem on Riemannian manifolds of positive curvature operator. Proc. Japan Acad. 50 (1974), 301-302.

Department of Mathematics, University of California at San Diego, La Jolla, CA 92093

E-mail address: lni@math.ucsd.edu

Department of Mathematics, University of California at San Diego, La Jolla, CA 92093

E-mail address: nwallach@math.ucsd.edu 